\title{
Cardiac Point-of-Care Ultrasound Publication Trends
}

\author{
Andrew Pattock ${ }^{1}$, Michelle Kim ${ }^{2}$, Cooper Kersey ${ }^{1}$, Linda Liu ${ }^{1}$, James Kirkpatrick ${ }^{1}$, \\ Adeyinka Adedipe ${ }^{1}$, Ross Kessler ${ }^{1}$, Amy Morris ${ }^{1}$, Sara Nikravan ${ }^{1}$, Sula Mazimba ${ }^{2}$, and \\ Younghoon Kwon ${ }^{1}$ \\ ${ }^{1}$ University of Washington \\ ${ }^{2}$ University of Virginia
}

October 1, 2021

\begin{abstract}
Introduction: Cardiac point-of-care ultrasound (c-POCUS) is an increasingly implemented diagnostic tool with the potential to guide clinical management. We sought to characterize and analyze the existing c-POCUS literature with a focus on the temporal trends and differences across specialties. Methods: A literature search for c-POCUS and related terms was conducted using Ovid (MEDLINE and Embase) and Web of Science databases through 2020. Eligible publications were classified by publication type and topic, author specialty, geographical region of senior author, and journal specialty. Results: The initial search produced 1761 potential publications. A strict definition of c-POCUS yielded a final total of 574 cardiac POCUS manuscripts. A yearly increase in c-POCUS publications was observed. Nearly half of publications were original research $(48.8 \%)$ followed by case report or series (22.8\%). Most publications had an emergency medicine senior author (37.5\%), followed by cardiology (20.2\%), anesthesiology $(12.2 \%)$, and critical care $(12.2 \%)$. The proportion authored by emergency medicine and cardiologists has decreased over time while those by anesthesiology and critical care has generally increased, particularly over the last decade. First authorship demonstrated a similar trend. Articles were published at similar numbers in emergency medicine journals (23.0\%) and cardiology journals (19.9\%). Conclusion: The annual number of c-POCUS publications has steadily increased over time reflecting the increased recognition and utilization of c-POCUS. This study can help inform clinicians of the current state of c-POCUS and augment the discussion surrounding barriers to continued adoption across all specialties.
\end{abstract}

\section{Hosted file}

Cardiac POCUS Manuscript Final.docx available at https://authorea.com/users/438919/articles/ 539985-cardiac-point-of-care-ultrasound-publication-trends 

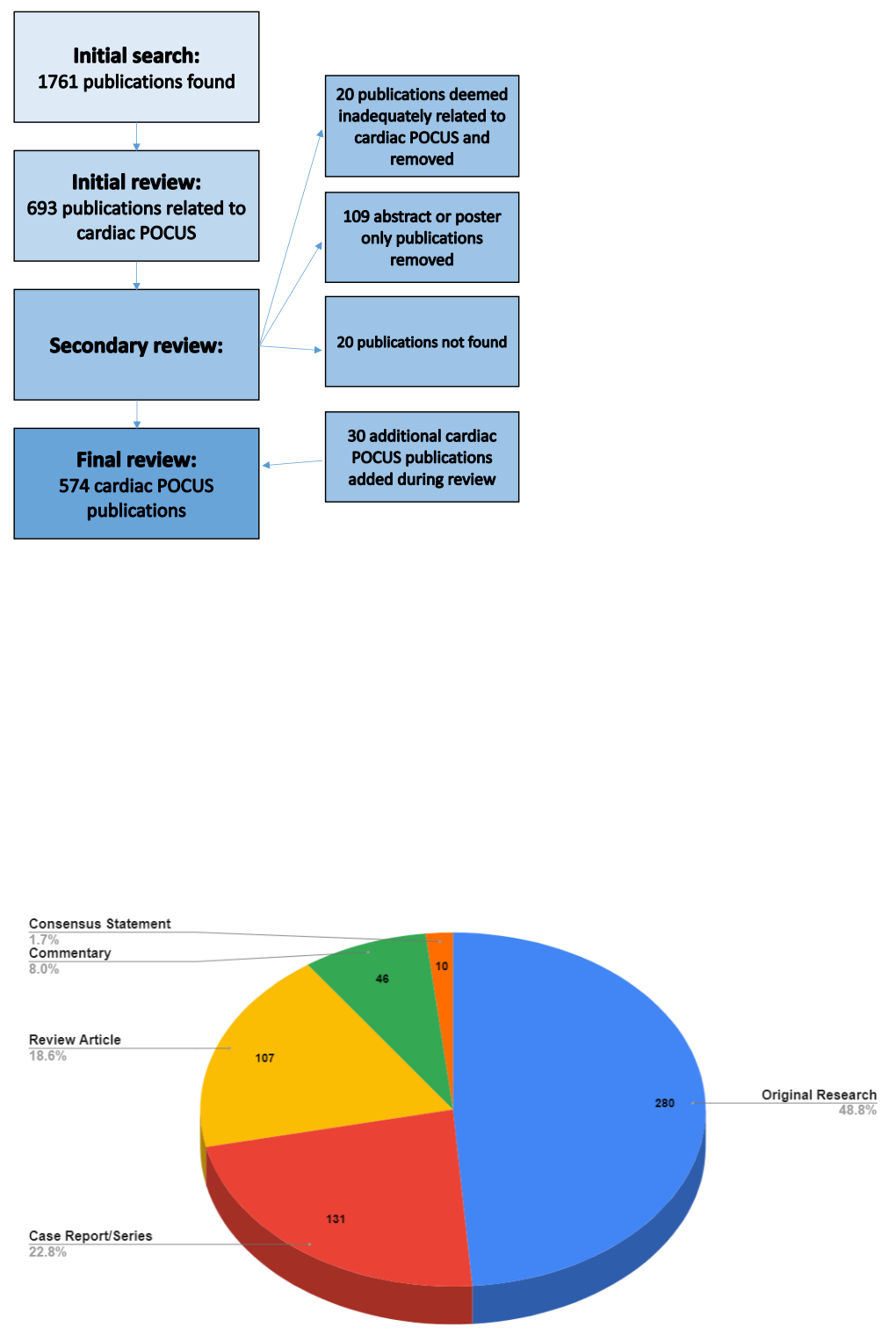

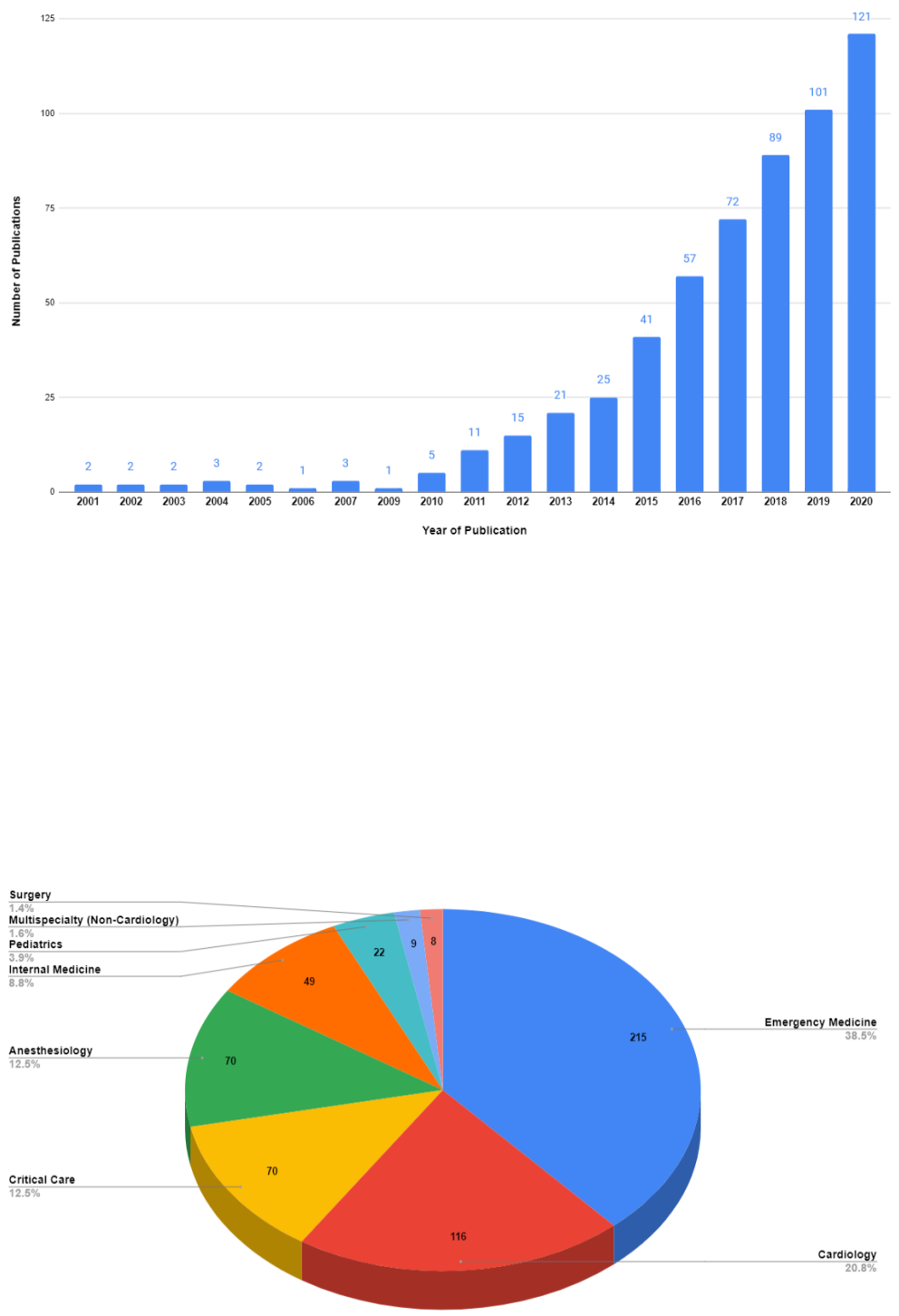

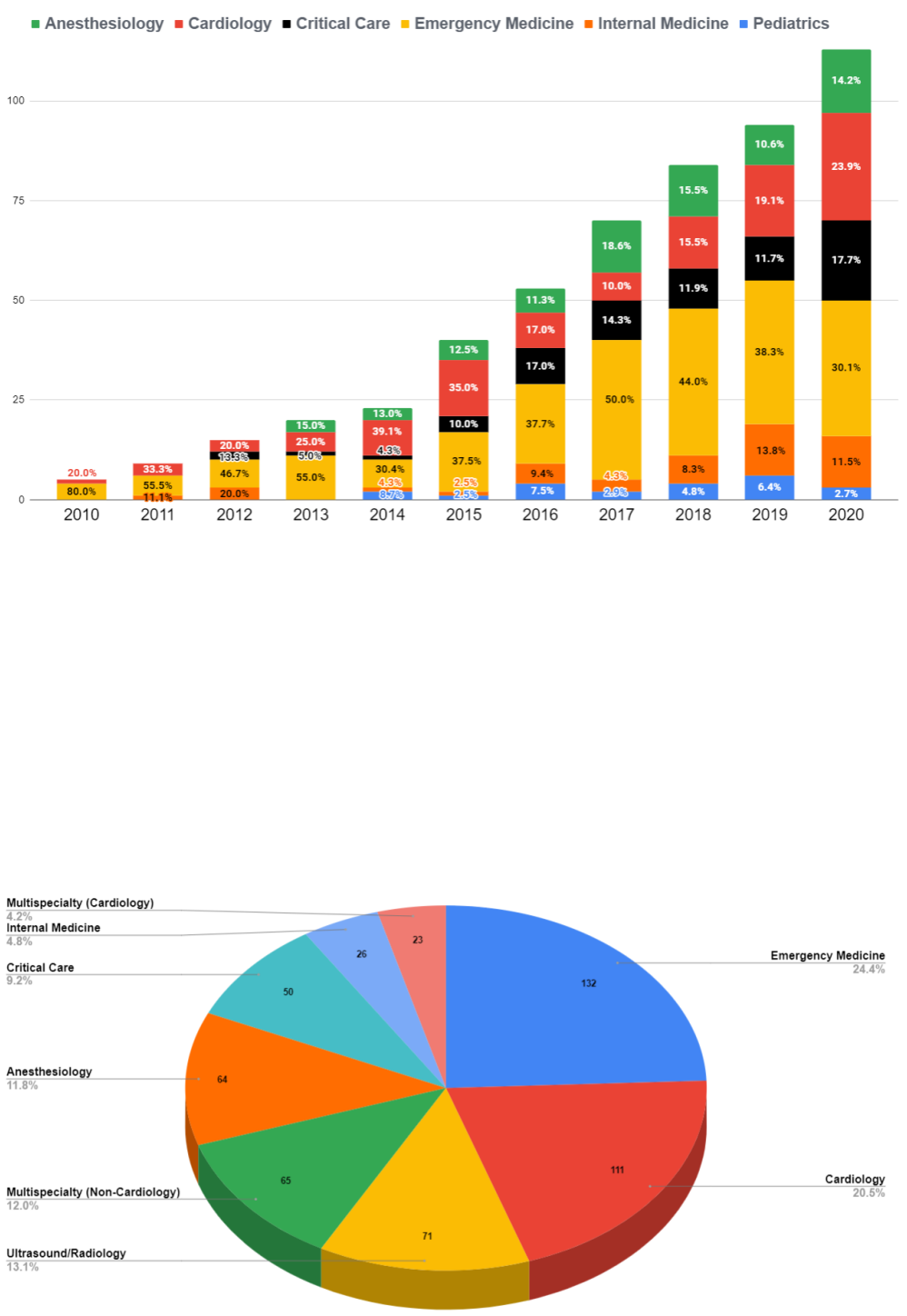


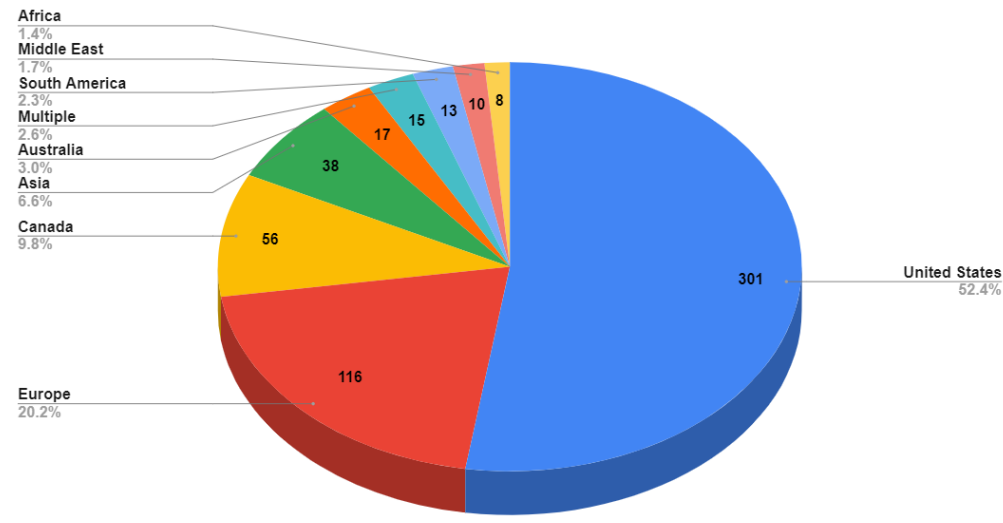

\begin{tabular}{lcc}
\multicolumn{1}{c}{ Article Topic Category } & Publications & Percent \\
Cardiovascular structure and function & 186 & $32.40 \%$ \\
Indications, perceptions, and efficacy & 185 & $32.23 \%$ \\
Volume status and shock & 66 & $11.50 \%$ \\
Cardiac arrest and resuscitation & 65 & $11.32 \%$ \\
Medical education and curriculum development & 58 & $10.10 \%$ \\
Other & 14 & $2.44 \%$ \\
Total & 574 & \\
\hline
\end{tabular}

